\title{
CT Perfusion Spot Sign Improves Sensitivity for Prediction of Outcome Compared with CTA and Postcontrast CT
}

\author{
A. Koculym, T.J. Huynh, R. Jakubovic, L. Zhang, and R.I. Aviv
}

\begin{abstract}
BACKGROUND AND PURPOSE: Recent studies have recommended both early and late imaging to increase spot sign detection. However optimal acquisition timing for spot detection and impact on outcome prediction is uncertain. Our aim was to assess the utility of CTP in spot sign detection and characterization with emphasis on its impact on the prediction of outcome in patients with acute primary $\mathrm{ICH}$.
\end{abstract}

MATERIALS AND METHODS: A retrospective review of 28 patients presenting within 6 hours of ICH, studied with CTA, CTP, and postcontrast CT, was performed. CTA, CTP, and postcontrast CT spot sign characteristics were recorded according to predefined radiologic criteria. A combined primary outcome of hematoma expansion or poor clinical outcome was used and defined as hematoma expansion $\geq 6 \mathrm{~mL}$ or $\geq 30 \%$, need for surgical drainage, or in-hospital mortality. Associations with the primary outcome and spot sign presence were examined against baseline clinical, laboratory, and radiographic variables. Predictive ability of CTA, CTP, and postcontrast CT spot characteristics were compared among modalities.

RESULTS: Primary outcome criteria were met in 18 patients (61\%). CTP spot sign presence was an independent predictor of hematoma expansion or poor outcome $(P=.040)$ and demonstrated greater sensitivity $(78 \%)$ than spots detected on CTA $(44 \%, P=.034)$ and postcontrast CT $(50 \%, P=.025)$. Specificity and positive predictive value of the spot sign was high (100\%) on all modalities. CTP detected the greatest number of spots ( $80 \%$ ) with peak spot attenuation demonstrated at a median (interquartile range) time of 50 seconds (range, 34-63 seconds) after contrast bolus injection. CTP spot appearance was later than CTA-detected spots $(P=.002)$ and earlier than postcontrast CT spots $(P<.001)$.

CONCLUSIONS: CTP spot sign detection improves the sensitivity for prediction of outcome compared with CTA or postcontrast CT-detected spots.

ABBREVIATIONS: $\mathrm{Cl}=$ confidence interval; $\mathrm{ICH}=$ intracerebral hemorrhage; $\mathrm{IVH}=$ intraventricular hemorrhage; $\mathrm{mRS}=$ modified Rankin Scale; $\mathrm{PCT}=$ postcontrast $\mathrm{CT}$

$\mathbf{P}$ rimary $\mathrm{ICH}$ occurs in $10 \%-30 \%$ of patients with stroke and is the most deadly and disabling type of stroke. ${ }^{1,2}$ Early hematoma expansion is an independent predictor of neurologic deterioration and mortality. ${ }^{3,4}$ Visualization of contrast extravasation within a hematoma on CTA, the spot sign, has been previously

Received May 4, 2012; accepted after revision August 6.

From the Department of Medical Imaging, University of Toronto and Sunnybrook Health Sciences Centre, Toronto, Ontario, Canada.

Paper previously presented in part at: 75th Anniversary of the Canadian Association of Radiologists Annual Scientific Meeting, April 26-29, 2012; Montreal, Quebec, Canada.

Please address correspondence to Richard I. Aviv, MD, Division of Neuroradiology, Department of Medical Imaging, Sunnybrook Health Sciences Centre, 2075 Bayview Ave, Room AG 31, Toronto, ON, M4N 3M5, Canada; e-mail: richard.aviv@ sunnybrook.ca

三 Indicates article with supplemental on-line table.

http://dx.doi.org/10.3174/ajnr.A3338 characterized and identified as an independent predictor of early hematoma expansion. ${ }^{5,6}$

A CTA spot sign score derived from the number, attenuation, and size of spots demonstrates an increased risk of hematoma expansion with increasing score. ${ }^{7}$ An inherent limitation of the spot sign score is that optimal CTA timing for spot visualization is unknown. Prior studies have demonstrated that delayed contrast imaging detects an additional $8 \%$ of spots not seen on early imaging. ${ }^{8-10}$ Furthermore, a prospective mulitcenter study using only early CTA for spot characterization demonstrated that $22 \%$ of spot-negative patients underwent hematoma expansion, ${ }^{6}$ likely underestimating the true number of patients with contrast extravasation. A large number of potential physiologic processes may affect spot sign visualization. Although rates of contrast injection, contrast dose, and volume can be standardized, there are varying blood pressure, circulation time, and perihematoma intracranial 
pressure changes that influence the time to spot visualization. It is, therefore, unlikely that a single optimal CTA time for spot sign delineation exists.

Recent studies have recommended both early and late imaging to increase spot detection, but there are no published data indicating that such studies will capture all foci of extravasation. CTP is a dynamic study that tracks a contrast bolus through the intracranial circulation typically for 60-120 seconds with modern biphasic techniques. CTP circumvents the need for early and late acquisitions and may show contrast extravasation not present on either CTA or PCT, reflecting the transient dynamics of spot sign opacification. ${ }^{11}$ Thus, it is likely that more spots may be detected with a dynamic study than with CTA or postcontrast imaging alone. Furthermore, peak opacification and extravasation size of the spot sign may occur at intervals later than those seen with traditional CTA. CTP may, therefore, also potentially improve the accuracy of the spot sign score for outcome.

The aim of this study was to assess the utility of CTP source images for spot sign detection and characterization, with emphasis on its impact on prediction of hematoma expansion or poor clinical outcome. We hypothesized that CTP would improve spot sign detection and demonstrate improved outcome prediction over CTA- or PCT-detected spots.

\section{MATERIALS AND METHODS \\ Patient Cohort}

This study was approved by the local institutional ethics review board. During a 6-month period, 28 consecutive patients with spontaneous ICH on NCCT, presenting to our tertiary stroke center $<6$ hours from ictus and consenting to baseline CTA, PCT, and CTP studies, were included in the study. Patients with secondary ICH (eg, suspected trauma, underlying aneurysm, vascular malformation, hemorrhagic conversion of ischemic stroke, venous sinus thrombosis, tumor, infection, or iatrogenic in-hospital procedure or illness) were excluded. Recruitment was performed during daytime hours because of the need for consent from the patient (or more commonly from the legal decision-maker) for the additional CTP scan. All 28 patients approached for inclusion into the study consented to the research protocol. When patients were enrolled, we explained that they would only be eligible for the CTP protocol after CTA was reviewed acutely to exclude secondary ICH. No secondary ICH causes occurred, and all consenting patients were included in the analysis.

\section{Clinical Data}

Baseline clinical variables were recorded in an ICH data base at ictus, and any missing data were retrieved by retrospective chart review. Data collected included patient demographics, medical history (anticoagulation, prior stroke, hypertension, diabetes, smoking, dyslipidemia, and alcohol abuse), time of symptom onset, baseline neurologic examination (NIHSS), blood pressure, and admission blood work (complete blood count, coagulation profile, and serum glucose). In-hospital mortality, need for surgical drainage, and discharge $\mathrm{mRS}$ scores were obtained from chart review by an experienced neurologist blinded to radiologic data.

\section{Imaging Acquisition}

All CT acquisitions were performed on a 64-section CT scanner (LightSpeed Plus and VCT; GE Healthcare, Milwaukee, Wisconsin). Standard baseline ICH evaluation at our institution includes a baseline NCCT, CTA, and PCT followed by a 24-hour NCCT. NCCT and PCT examinations were performed from the skull base to the vertex with the following parameters: $120 \mathrm{kV}$ (peak), 340 $\mathrm{mA}, 4 \times 5 \mathrm{~mm}$ collimation, 1 s/rotation, and a table speed of 15 $\mathrm{mm} /$ rotation. CTA studies were acquired from C6 to the vertex in the helical half-scan mode with the following parameters: 0.7 $\mathrm{mL} / \mathrm{kg}$ of iodinated contrast (to a maximum of $90 \mathrm{~mL}$ through an antecubital vein via at least an 18- or 20-ga angiocatheter), 120 $\mathrm{kV}(\mathrm{p}), 270 \mathrm{~mA}, 1 \mathrm{~s} /$ rotation, $1.25-\mathrm{mm}$ section thickness at $0.625-\mathrm{mm}$ intervals, and table speed of $3.75 \mathrm{~mm} /$ rotation. CTA contrast bolus timing was obtained by using a SmartPrep (GE Healthcare) semiautomated attenuation-triggered technique. A biphasic CTP study was added at baseline after consent was obtained. The first phase was a 45 -second continuous (cine) scan reconstructed at 0.5 -second intervals to produce a series of 90 sequential images for each of the 8 sections, covering a total of 40 $\mathrm{mm}$. The second phase collected images covering the same $8 \mathrm{sec}-$ tions during an additional 90 seconds, immediately after the first phase at intervals of 15 seconds. Scan parameters for both phases were the following: $80 \mathrm{kV}(\mathrm{p}), 190 \mathrm{~mA}, 8 \times 5 \mathrm{~mm}$ collimation, and 1 s/rotation. On the basis of the NCCT findings, CTP was targeted to provide the largest hematoma coverage possible. Iodinated contrast (dose, $0.5 \mathrm{~mL} / \mathrm{kg}$; maximum, $50 \mathrm{~mL}$; rate, $4 \mathrm{~mL} / \mathrm{s}$ ) was injected 3-5 seconds before the start of the first CTP phase.

\section{Imaging Analysis}

All scans were reviewed jointly by 2 readers with a third experienced neuroradiologist providing final consensus. Spots signs were defined as foci of contrast extravasation within the hematoma with attenuation $>120 \mathrm{HU}$ and were visualized on spot windows (width, 200; level, 100). The total number of spots, maximum spot attenuation, and axial dimensions were recorded for all patients on each of the CTA, CTP, and PCT source images. Each spot was numbered sequentially on each study, and annotations were saved to the PACS. Each technique was reviewed blinded to the others with a 1-week interval in between to avoid recall bias. After the 3 reviews, each patient was again examined to cross-reference spots among imaging modalities to determine whether they were present on the other study types. A cross-reference tool was used to determine the status of each previously labeled spot. Spot sign scores were calculated for each technique as previously published, considering maximum axial dimension, attenuation, and number of spots. ${ }^{7}$ Time from CTP contrast bolus injection to maximal spot attenuation was recorded in seconds for CTP spots. Time from contrast bolus injection to CTA and PCT acquisition was obtained from archived SmartPrep images (GE Healthcare). Baseline and 24-hour follow-up NCCT ICH volumes were calculated by using the $\mathrm{ABC} / 2$ method. ${ }^{12}$ Hematoma expansion was defined as an increase in volume of $>6 \mathrm{~mL}$ or $>30 \% .{ }^{8}$ The presence of IVH and Graeb score ${ }^{13}$ on baseline imaging was measured; however, it was not considered in the calculation of hematoma expansion. Ultra-early hematoma growth was calculated by dividing the initial hematoma volume by the 
hours from ictus onset. ${ }^{14}$ Studies were viewed on PACS workstations.

\section{Statistical Analysis}

A combined primary outcome of hematoma expansion or poor clinical outcome was used and defined as hematoma expansion, need for emergent surgical drainage, or in-hospital mortality. Patients undergoing emergent surgery were included in the primary outcome because these patients often demonstrate early clinical deterioration, prompting surgery, and have poor clinical outcomes. ${ }^{15}$ Univariate associations with the primary outcome and spot sign presence were examined against baseline clinical, laboratory, and radiographic variables. Continuous data were assessed by using the Student $t$ test or Wilcoxon rank-sum test on the basis of the normality of data by using the Shapiro-Wilk test. Categoric data were assessed by using the Fisher exact test. Multivariable logistic regression for primary outcome prediction was performed including all variables with $P<.10$ on univariate analysis. Firth bias-correction was used to account for complete separation of data. ${ }^{16}$ Model calibration was assessed with the Hosmer-Lemeshow test. Individual spot sign characteristics on CTA, CTP, and PCT were also compared against the primary combined outcome. The predictive ability of spot properties by imaging technique was assessed by using receiver operating characteristic curve analyses. Diagnostic performance for prediction of poor outcome and spot detection between imaging techniques was performed by using ROC curve comparison and the McNemar test for continuous and dichotomous variables, respectively. The Kruskal-Wallis test was used to examine differences in spot characteristics between modalities overall. Statistical associations with $P \leq .05$ were considered significant. All statistical analyses were performed in SAS, Version 9.2 (SAS Institute, Cary, North Carolina) and R, Version 2.13.2 (http://www.r-project.org/).

\section{RESULTS}

Patient characteristics and univariate associations with the primary combined outcome are summarized in the On-line Table. Primary outcome criteria were met by 18 patients (61\%), with 11 (39\%) patients dying in-hospital (3 before 24-hour follow-up CT), 6 (21\%) undergoing surgical hematoma evacuation before follow-up CT, and 6 (21\%) exhibiting hematoma expansion. Of the 6 patients undergoing surgery, 3 (50\%) died in-hospital and the remainder experienced moderate-to-severe disability (mRS score $\geq 4)$. Of the 6 patients with hematoma expansion, $2(33 \%)$ died in-hospital and $2(33 \%)$ were left with moderate-to-severe disability (mRS score $\geq 4$ ). Primary outcome was significantly associated with greater median baseline NIHSS score, IVH Graeb score, initial and final hematoma volume, ultra-early hematoma growth, percentage hematoma expansion, and spot presence on all imaging techniques $(P<.05)$. Patients with the primary outcome also had a trend toward increased absolute hematoma expansion $(P=.053)$. Multivariable regression demonstrated that CTP spot presence was the only independent predictor of the primary outcome after adjusting for initial ICH volume, ultraearly hematoma growth, NIHSS score, and IVH Graeb score (odds ratio, 13.7; 95\% CI, 1.12-166; $P=.040$ ).

Greater median (interquartile range) initial hematoma vol- ume was associated with spot presence on CTA (104 mL [interquartile range, $70.3-160 \mathrm{~mL}$ ] versus $10.7 \mathrm{~mL}$ [interquartile range, 2.9-32.4 mL]; $P=.008$ ), CTP (102 mL [interquartile range, 15.1$130 \mathrm{~mL}$ ] versus $3.4 \mathrm{~mL}$ [interquartile range, $2.3-59.9 \mathrm{~mL}$ ]; $P<$ $.001)$, and PCT (106 mL [interquartile range, $16.0-131 \mathrm{~mL}$ ] versus $9.7 \mathrm{~mL}$ [interquartile range, $2.8-36.2 \mathrm{~mL}$ ]; $P=.007)$. CTP spot presence was significantly associated with greater median ultra-early hematoma growth $(25.5 \mathrm{~mL} / \mathrm{h}$ [interquartile range, $15.6-41.2 \mathrm{~mL} / \mathrm{h}$ ] versus $2.0 \mathrm{~mL} / \mathrm{h}$ [interquartile range, $1.0-11.5$ $\mathrm{mL} / \mathrm{h}$ ]; $P=.012$ ), final hematoma volume ( $48.5 \mathrm{~mL}$ [interquartile range, $22.2-108.0 \mathrm{~mL}$ ] versus $3.2 \mathrm{~mL}$ [interquartile range, $1.9-$ $10.8 \mathrm{~mL}] ; P=.009)$, absolute hematoma expansion volume (7.9 $\mathrm{mL}$ [interquartile range, $7.0-8.3 \mathrm{~mL}$ ] versus $0.1 \mathrm{~mL}$ [interquartile range, $-1.0-0.2 \mathrm{~mL}] ; P=.017)$, and in-hospital mortality $(64 \%$ versus $14 \% ; P=.018$ ) with a trend toward a greater percentage of hematoma expansion $(46.3 \%$ [interquartile range, $15.1 \%$ $62.8 \%$ ] versus $1.6 \%$ [interquartile range, $-10.3 \%-10.3 \%$ ], $P=$ .058). A higher baseline NIHSS score was noted among CTP (23 [interquartile range, 20-26] versus 12 [interquartile range, 3-15]; $P=.002)$ and PCT-spot-positive patients (27 [interquartile range, 23-33] versus 19 [interquartile range, $14-24$ ]; $P=.006$ ).

\section{Outcome Prediction Performance by Imaging Technique}

Fifteen (54\%) patients demonstrated a spot sign with 8 (29\%), 14 (50\%), and 9 (32\%) spot-positive patients found on CTA, CTP, and PCT, respectively. The corresponding sensitivity for the prediction of primary outcome was 44\% (95\% CI, 22\%-69\%), 78\% (95\% CI, 52\%-94\%), and 50\% (95\% CI, 26\%-74\%), respectively, for each CT technique. CTP spot sensitivity was significantly greater than CTA $(P=.034)$ and PCT $(P=.025)$ spots. Specificity and positive predictive value for primary outcome prediction was $100 \%$ for the presence of spot signs on all modalities (95\% CI, CTA: $69 \%-100 \%$, 63\%-100\%; CTP: 69\%-100\%, 77\%-100\%; PCT: $69 \%-100 \%, 66 \%-100 \%$, respectively). Negative predictive value for CTA, CTP, and PCT was 50\% (95\% CI, 27\%-73\%), 71\% (95\% CI, 42\%-92\%), and 53\% (95\% CI, 29\%-76\%), respectively. All PCT spot-positive patients were identified as spot-positive on CTP. With the exception of a single patient $(1 / 8 ; 13 \%)$, all CTA spot-positive patients were classified as spot-positive on CTP. The single spot-positive patient who was missed on CTP was due to inadequate spatial coverage through the hematoma. A combination of spot presence on CTA or PCT improved the sensitivity for primary outcome prediction to $61 \%(95 \% \mathrm{CI}, 36 \%-83 \%)$ and had a trend toward improved sensitivity over CTA spot presence alone $(P=.083)$. This however was not significantly improved over PCT spot sensitivity $(P=.157)$. CTP spot positivity had the highest sensitivity for primary outcome prediction, detecting 3 additional true-positive patients compared with combined CTA or PCT, though this did not reach statistical significance $(P=$ .180). Comparison between CTA and PCT spot-positive patients demonstrated that $2(7 \%)$ patients were true-positive only on CTA and 3 (11\%) patients were true-positive only on PCT. There was, however, no statistical difference between CTA and PCT spot sensitivity for the prediction of the combined outcome $(P=$ $.655)$.

AJNR Am J Neuroradiol 34:965-70 May 2013 www.ajnr.org 967 


\section{Spot Characteristics by Imaging Technique}

Radiologic characteristics of the spot sign by imaging technique are summarized in the Table. Overall maximum spot attenuation varied among modalities $(P=.011)$, with PCT spots significantly less attenuated than CTA $(P=.009)$ or CTP $(P=.008)$ spots. No difference was noted between maximum CTA and CTP spot densities $(P=.868)$. The maximum axial dimension was significantly larger among PCT spots compared with CTA spots $(P=.018)$. No differences in maximum axial dimensions were noted between CTP and PCT spots $(P=.369)$; however, there was a trend toward increased CTP spot size compared with CTA $(P=.094)$. The time from contrast bolus injection to maximum spot attenuation was significantly longer for CTP compared with CTA $(P=.002)$ and significantly shorter than PCT $(P<.001)$. Patients classified as CTA spot-negative who were positive on CTP $(n=7)$ tended to have fewer CTP spots than those positive on both CTA and CTP $(n=7)$ (1 [range, 1-2] versus 4 [range, 2-6] $P=.058$ ).

Primary outcome was associated with the spot number on CTA $(P=.017)$, CTP $(P<.001)$, and PCT $(P=.010)$, with a corresponding area under the curve of 0.722 (95\% CI, 6040.840), 0.889 (95\% CI, 0.790-0.988), and 0.750 (95\% CI, 0.6310.869 ), respectively. Analysis of receiver operating characteristic curves demonstrated that maximal specificity was obtained by spot presence or absence on each technique; and further stratification by number, attenuation, size, and spot sign score did not yield additional predictive performance benefit.

Analysis of individual spots on each technique independently demonstrated 28, 51, and 17 spots on CTA, CTP, and PCT, respectively. After we cross-referenced spot location among modalities, there were a total of 70 possible spots identified. CTP detected the greatest number of spots $(56 / 70,80 \%)$ compared with CTA $(28 / 70,40 \% ; P<.001)$ and PCT $(21 / 70,30 \%$; $P<.001)$. A combination of spots identified on CTA or PCT improved overall spot detection $(39 / 70,56 \%)$; however, CTP remained significantly better for spot detection $(P=.011)$. If we examined spots not seen among modalities, 18 (64\%) and 10 (36\%) CTA spots were found to have not been visualized on PCT and CTP, 38 (75\%) and 39 (76\%) CTP spot foci were not visualized on CTA and PCT, and 11 (65\%) and 5 (29\%) PCT spots were not visualized on CTA and CTP, respectively. An analysis of CTP spots not visualized on CTA demonstrated that spots missed on CTA had a greater time from contrast bolus injection to maximum attenuation (44 seconds [33-105 seconds] versus 27 seconds [24-33 seconds] $P=.014)$ and were also smaller $(6.5 \mathrm{~mm}$ [interquartile range, $5.0-9.9 \mathrm{~mm}$ ] versus $4.4 \mathrm{~mm}$ [interquartile range, 3.5-6.4 $\mathrm{mm}] ; P=.026)$ compared with CTP spots seen on CTA. CTP spots not visualized on PCT were significantly less attenuated (311 HU [interquartile range, 203-372 HU] versus $156 \mathrm{HU}$ [interquartile range, $148-190 \mathrm{HU}], P<.001)$ and smaller $(4.3 \mathrm{~mm}$ [interquartile range, $3.4-5.8 \mathrm{~mm}$ ] versus $16.8 \mathrm{~mm}$ [interquartile range, $6.0-24.5 \mathrm{~mm}$ ], $P<.001)$ than CTP spots seen on PCT.
CTA and PCT spots not detected on CTP could all be attributed to incomplete hematoma coverage.

\section{DISCUSSION}

The use of CTP source images for spot sign detection resulted in greater spot sign and spot-positive patient detection compared with CTA and PCT. CTP spot sign independently predicted hematoma expansion or poor clinical outcome and was also significantly associated with greater absolute hematoma expansion volume, final hematoma volume, and in-hospital mortality. The increased sensitivity for spot detection was largely attributed to a delay in the appearance of maximal spot attenuation at 30-70 seconds, appearing later than the traditional CTA acquisition time of 20-26 seconds (Fig 1). Increased sensitivity over PCT was also due to the detection of smaller and less attenuated spots that dispersed or became subthreshold attenuation on PCT at 300360 seconds. These findings highlight the disadvantages of single arbitrarily timed CTA acquisitions for the detection of extravasation, which may limit accurate prediction of hematoma expansion and poor outcome. These findings also reinforce the observations by Chakraborty et $\mathrm{al}^{11}$ and emphasize the critical timesensitive nature of spot sign appearance.

The presence or absence of the spot sign was the best spot characteristic for prediction of hematoma expansion or poor outcome, demonstrating maximal sensitivity and specificity for all modalities. These results precluded any study of predictive value improvement with the spot sign score. Similarly, previous studies of CTA spot sign performance have demonstrated specificities between $80 \%$ and $100 \%$ for hematoma expansion. ${ }^{5-7,10}$ We observed a positively skewed distribution of initial hematoma volumes with small initial volumes in patients not meeting the combined outcome. Smaller hematomas are less likely to harbor underlying contrast extravasation and undergo expansion. Supporting this finding is a prior study demonstrating that smaller initial hematomas $(<10 \mathrm{~mL})$ uncommonly undergo hematoma expansion or experience poor outcome. ${ }^{17}$ Furthermore, an association between larger baseline hematoma and contrast extravasation is shown. ${ }^{6}$ The high reported specificity in this series may also be the result of the used threshold proposed by Delgado Almandoz et $\mathrm{al}^{7}$ as a cutoff for spot sign definition. Although the threshold was selected to facilitate spot sign detection from background hematoma attenuation, the delay to peak attenuation shown with CTP indicates that while this threshold improves CTA specificity, sensitivity is reduced. The optimal threshold selection for early CTA remains uncertain and is dependent on both the rate of leakage and scan timing. Although formal validation is 

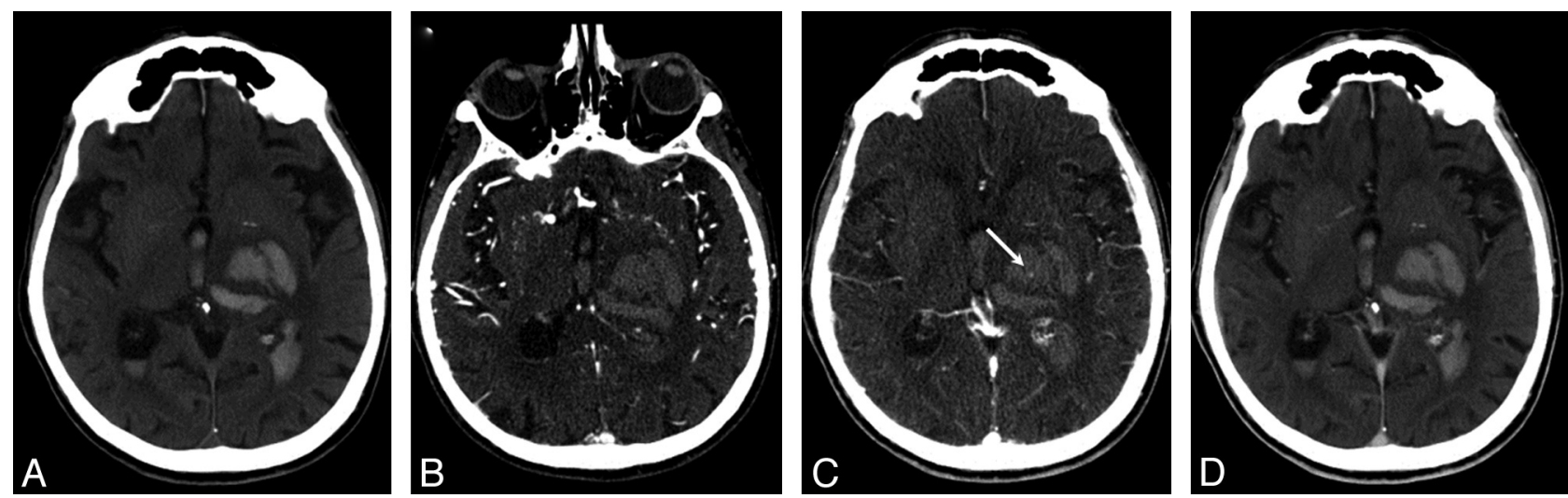

FIG 1. A, NCCT demonstrates an ICH, with IVH extension, without evidence of calcification within. $B$, No spot signs are detected on CTA. C, CTP identifies a 2-mm spot sign (arrow) with maximum attenuation appearing 40 seconds after contrast bolus. $D$, PCT demonstrates no definite contrast extravasation. The patient clinically deteriorated rapidly and died before 24-hour CT.

required, we have observed that an attenuation of $>100 \mathrm{HU}$ may be a more sensitive threshold for spot identification.

Following the mixed results of the phase 2 and 3 acute ICH recombinant activated factor VII (rFVIIa) therapy trials, ${ }^{18,19}$ there has been growing interest in the CTA spot sign as a predictor of hematoma expansion to facilitate targeted hemostatic therapy. ${ }^{20-22}$ CTP source image use increases the sensitivity for spot detection and, more important, the sensitivity for prediction of hematoma expansion or poor clinical outcome while maintaining high specificity. When CTP was used for spot sign screening, $50 \%$ of patients demonstrated a spot sign compared with $29 \%$ detected on CTA. A prevalence of $29 \%$ is concordant with prior reports. ${ }^{5-7}$ These results strongly suggest that early CTA may not be sufficient to fully define the ICH population at risk for hematoma expansion. This deficiency may critically affect trials recruiting patients for rFVIIa, not only because of the relatively large sample required to achieve sufficient spot-positive patients but also due to misclassification of patients as spot-negative. Such concern was recently raised by Wardlaw ${ }^{23}$ commenting on a $22 \%$ rate of hematoma growth in early CTA-designated spot-negative patients in a prospective ICH study. ${ }^{6} \mathrm{CTP}$ source images identify a greater number of patients who are at risk for poor outcome and who may potentially benefit from therapeutic intervention. Our results confirm that detection of more patients who are actually spot-positive on CTP contributes to better outcome prediction than CTA and PCT alone. CTP therefore represents an ideal technique for extravasation imaging and may improve patient selection for therapy.

The combination of CTA and PCT was shown to be inferior to CTP for detection of individual spots; however, it was not significantly worse for detection of spot presence in patients. Our study, however, had limited power to detect small differences in sensitivity. Furthermore, CTP does provide a longer time window to observe contrast extravasation and the additional benefit of quantification of the rate of extravasation. ${ }^{24}$ Radiation doses from NCCT, CTA, CTP, and PCT were 2.6, 3.5, 2.5, and $2.6 \mathrm{mSv}$, respectively. The temporal resolution of CTP can be reduced to address any dose concerns (or facilitate greater spatial coverage) because the higher temporal resolution required for perfusion imaging is not necessary for spot detection. We propose that a uniphasic sequence with 15-second resolution be used for a 2-minute total duration. Reducing the spatial coverage to the region of interest through the hematoma and angulation to avoid the orbits is expected to significantly reduce the CTP dose to less than that of a conventional NCCT or CTA. The protocol would also significantly reduce the heterogeneity of the observed prevalence and characterization of contrast extravasation currently reported with PCT and early and delayed CTA imaging. ${ }^{8-10}$ Given the potential improvement in diagnostic efficacy, extra, modest radiation exposure for improved diagnostic efficacy may outweigh any risk associated with stochastic effects. Additionally older patients who typically constitute the ICH population have a lower risk of a stochastic effect.

An important limitation of this study was inadequate spatial coverage of hematoma volume by CTP. In 1 patient, this resulted in misclassification of a CTA spot-positive patient. It is possible that more spots and spot-positive patients would have been identified if the entire hematoma had been visualized. Techniques to increase spatial coverage included table toggle techniques, ${ }^{25} \mathrm{CTP}$ repeated at 2 consecutive levels, ${ }^{26}$ and the use of 320 -section scanners with inherent greater spatial coverage. ${ }^{27,28}$ Our study has a small sample size, reflecting the difficulty in recruiting patients with ICH into hyperacute-phase imaging studies. Validation of our findings in a larger prospective sample is thus required.

\section{CONCLUSIONS}

Use of CTP source images in acute ICH improves the sensitivity for spot sign detection and prediction of hematoma expansion or poor clinical outcome over CTA and PCT alone. CTP-detected spots could increase the number of patients with acute ICH eligible for enrolment in trials of hemostatic therapy; however, these findings should be further validated in larger studies.

\section{REFERENCES}

1. Morgenstern LB, Hemphill JC Jr, Anderson C, et al. Guidelines for the management of spontaneous intracerebral hemorrhage: a guideline for healthcare professionals from the American Heart Association/American Stroke Association. Stroke 2010;41:2108-29

2. Russell MW, Joshi AV, Neumann PJ, et al. Predictors of hospital length of stay and cost in patients with intracerebral hemorrhage. Neurology 2006;67:1279-81

3. Brott T, Broderick J, Kothari R, et al. Early hemorrhage growth in patients with intracerebral hemorrhage. Stroke 1997;28:1-5

AJNR Am J Neuroradiol 34:965-70 May 2013 www.ajnr.org 
4. Leira R, Dávalos A, Silva Y, et al. Early neurologic deterioration in intracerebral hemorrhage: predictors and associated factors. $\mathrm{Neu}$ rology 2004;63:461-67

5. Wada R, Aviv RI, Fox AJ, et al. CT angiography “spot sign” predicts hematoma expansion in acute intracerebral hemorrhage. Stroke 2007;38:1257-62

6. Demchuk AM, Dowlatshahi D, Rodriguez-Luna D, et al. Prediction of haematoma growth and outcome in patients with intracerebral haemorrhage using the CT-angiography spot sign (PREDICT): a prospective observational study. Lancet Neurol 2012;11:307-14

7. Delgado Almandoz JE, Yoo AJ, Stone MJ, et al. Systematic characterization of the computed tomography angiography spot sign in primary intracerebral hemorrhage identifies patients at highest risk for hematoma expansion: the spot sign score. Stroke 2009;40:29943000

8. Ederies A, Demchuk A, Chia T, et al. Postcontrast CT extravasation is associated with hematoma expansion in CTA spot negative patients. Stroke 2009;40:1672-76

9. Delgado Almandoz JE, Yoo AJ, Stone MJ, et al. The spot sign score in primary intracerebral hemorrhage identifies patients at highest risk of in-hospital mortality and poor outcome among survivors. Stroke 2010;41:54-60

10. Hallevi H, Abraham AT, Barreto AD, et al. The spot sign in intracerebral hemorrhage: the importance of looking for contrast extravasation. Cerebrovasc Dis 2010;29:217-20

11. Chakraborty S, Blacquiere D, Lum C, et al. Dynamic nature of the CT angiographic “spot sign.” Br J Radiol 2010;83:e216-19

12. Kothari RU, Brott T, Broderick JP, et al. The ABCs of measuring intracerebral hemorrhage volumes. Stroke 1996;27:1304-05

13. Graeb DA, Robertson WD, Lapointe JS, et al. Computed tomographic diagnosis of intraventricular hemorrhage: etiology and prognosis. Radiology 1982;143:91-96

14. Rodriguez-Luna D, Rubiera M, Ribo M, et al. Ultraearly hematoma growth predicts poor outcome after acute intracerebral hemorrhage. Neurology 2011;77:1599-604

15. Rabinstein AA, Atkinson JL, Wijdicks EFM. Emergency craniotomy in patients worsening due to expanded cerebral hematoma: to what purpose? Neurology 2002;58:1367-72
16. Firth D. Bias reduction of maximum likelihood estimates. $B i$ ometrika 1993;80:27-38

17. Dowlatshahi D, Smith EE, Flaherty ML, et al. Small intracerebral haemorrhages are associated with less haematoma expansion and better outcomes. Int J Stroke 2011;6:201-16

18. Mayer SA, Brun NC, Begtrup K, et al. Recombinant activated factor VII for acute intracerebral hemorrhage. $N$ Engl J Med 2005;352:777-85

19. Mayer SA, Brun NC, Begtrup K, et al. Efficacy and safety of recombinant activated factor VII for acute intracerebral hemorrhage. N Engl J Med 2008;358:2127-37

20. Mayer SA, Davis SM, Skolnick BE, et al. Can a subset of intracerebral hemorrhage patients benefit from hemostatic therapy with recombinant activated factor VII? Stroke 2009;40:833-40

21. The Spot Sign for Predicting and Treating ICH Growth Study. http:// clinicaltrials.gov/ct $2 /$ results?term $=$ The + Spot + Sign + for + Predicting + and + Treating\&Search $=$ Search. Accessed July 25, 2012.

22. "Spot Sign" Selection of Intracerebral Hemorrhage to Guide Hemostatic Therapy (SPOTLIGHT): randomized controlled trial. ISRCTN29749408. http://clinicaltrials.gov/ct2/show/NCT00810888. Accessed July 25, 2012.

23. Wardlaw JM. Prediction of haematoma expansion with the CTA spot sign: a useful biomarker? Lancet Neurol 2012;11:294-95

24. d'Esterre CD, Chia TL, Jairath A, et al. Early rate of contrast extravasation in patients with intracerebral hemorrhage. AJNR Am J Neuroradiol 2011;32:1879-84

25. Youn SW, Kim JH, Weon YC, et al. Perfusion CT of the brain using 40-mm-wide detector and toggling table technique for initial imaging of acute stroke. AJR Am J Roentgenol 2008;191:W120-26

26. Wintermark M, Smith WS, Ko NU, et al. Dynamic perfusion CT: optimizing the temporal resolution and contrast volume for calculation of perfusion CT parameters in stroke patients. AJNR Am J Neuroradiol 2004;25:720-29

27. Murayama K, Katada K, Nakane M, et al. Whole-brain perfusion CT performed with a prototype 256-detector row CT system: initial experience. Radiology 2009;250:202-11

28. Yang CY, Chen YF, Lee CW, et al. Multiphase CT angiography versus single-phase CT angiography: comparison of image quality and radiation dose. AJNR Am J Neuroradiol 2008;29:1288-95 